\title{
NONLINEAR FATIGUE DAMAGE ACCUMULATION
}

\author{
Daniel Krzysztof Dębski \\ Warsaw University of Technology, Narbutta 84 Street, 02-524 Warsaw, Poland \\ daniel.debski@simr.pw.edu.pl
}

\begin{abstract}
One important element of any computational fatigue analysis is the adoption of a hypothesis of fatigue damage accumulation. The most commonly used is the hypothesis of linear accumulation of fatigue damage called the Palmgren-Miner hypothesis. This linear hypothesis does not take into account a factor of great importance: the mutual influence of consecutive fatigue load sequences on each other. In the presented paper, only two consecutive load sequences linked by mutual relations have been analyzed and the results of the analysis have been shown. A more complex form which takes into account the full load history would create complex formula difficult to use. Perhaps, we should go in this direction, especially that today we have enormous computing power at our disposal.
\end{abstract}

Keywords: cumulative fatigue damage; fatigue damage accumulation; cumulative damage rules; load interaction effects; fatigue life prediction.

\section{INTRODUCTION}

One essential element of any computational fatigue analysis is the adoption of a hypothesis of fatigue damage accumulation. The most commonly used is the hypothesis of linear accumulation of fatigue damage (1924), also called the Palmgren-Miner hypothesis, in honor of its authors [1, 2]. It assumes that the measure of assessment of the fatigue damage $D_{j}$, when $j$ fatigue load sequences were applied to the load-bearing structure, is a linear relationship:

$$
D_{j}=\sum_{i=1}^{j} \frac{n_{i}}{N_{i}}
$$

In this case, $n_{\mathrm{i}}$ is the number of load cycles applied to the load-bearing structure in the $i$-th sequence, while $N_{i}$ is a number of cycles of the load that causes fatigue damage to the structure. Experience has shown that the critical value of fatigue damage, also known as fatigue wear $D_{j}$, lies usually in the interval: $0.5 \leq D \leq 2.5$. In the fatigue analyses, a value of $D=1$ is usually assumed. The engineering practice has shown that it is usually a safe value in terms of structure fatigue assessment.

The linear hypothesis of fatigue damage accumulation does not take into account a factor of great importance: the mutual influence of consecutive fatigue load sequences on each other.

The figure below shows two spectra of loads $a$ and $b$.

From the perspective of the Palmgren-Miner hypothesis, the assessment of fatigue wear $D$ has the identical value in both cases:

$$
\stackrel{a)}{=} \frac{n_{1}}{N_{1}}+\frac{n_{2}}{N_{2}} \stackrel{b)}{=} \frac{n_{2}}{N_{2}}+\frac{n_{1}}{N_{1}}
$$




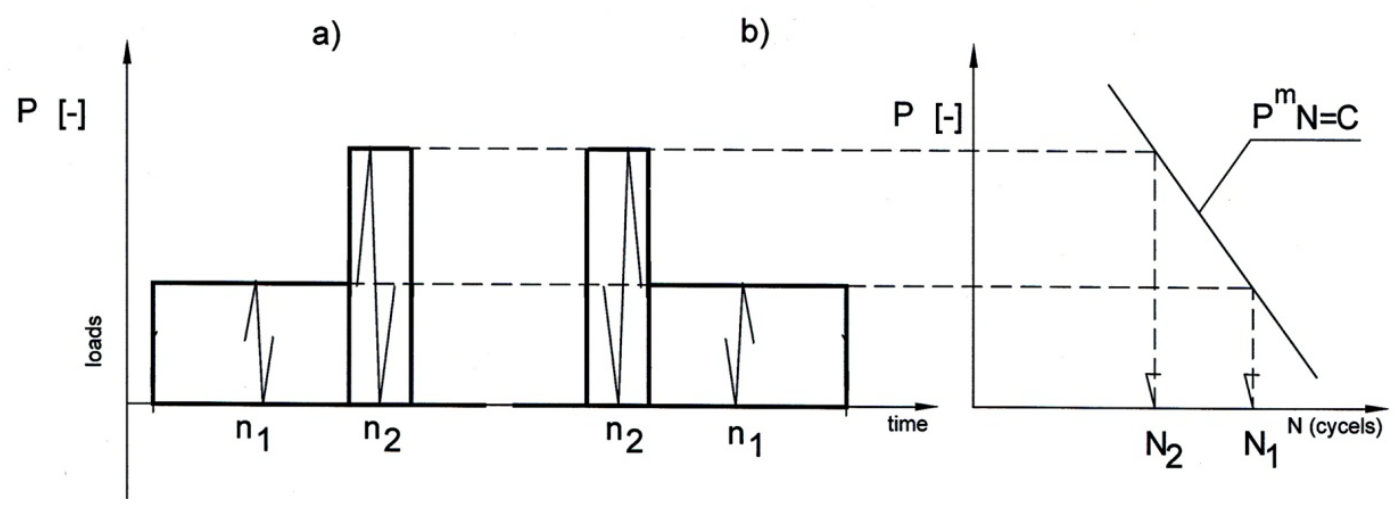

Figure 1. Examples of possible fatigue load sequences and an exemplary fatigue curve

In practice, one may expect a lower value of the fatigue wear for spectrum $b$ ) than for spectrum a). Due to the application of the load sequence: higher load - lower load, there may appear, in the areas of structural or material notches, the plastic deformation zones larger than for spectrum $a$ ), as early as in the initial load phase. They will effectively inhibit the development of fatigue damage caused by another sequence of smaller loads. This consequence is not reflected by the PalmgrenMiner hypothesis. There is no doubt that the mutual influence of consecutive fatigue load sequences has a significant impact on fatigue wear of the load-bearing structure.

The difference in value of the subsequent cycles of loads $P_{i-1}$ and $P_{i}$ generates the change of local loads in the area of developing fatigue damage. This can be described as a fatigue-equivalent change of global loads:

$$
P^{*}{ }_{i}=P_{i}-\alpha P_{i-1}
$$

where the coefficient - $\alpha$ is a measure of fatigue-equivalent changes of the aforementioned local loads in the area of fatigue damage, caused by the load of global $P_{i-1}$ preceding $P_{i}$. The value of the coefficient - $\alpha$ is chosen or arbitrarily assumed based on the results of fatigue tests. If, according to the above, a fatigue curve is described by the equation: $P_{i}^{m} N_{i}=C$, then the corrected number of cycles until destruction - $N_{i}^{*}$ (Fig. 2) will be described by the relation:

$$
N_{i}^{*}=\frac{N_{i}}{\left(1-\alpha \frac{P_{i-1}}{P_{i}}\right)^{m}}
$$

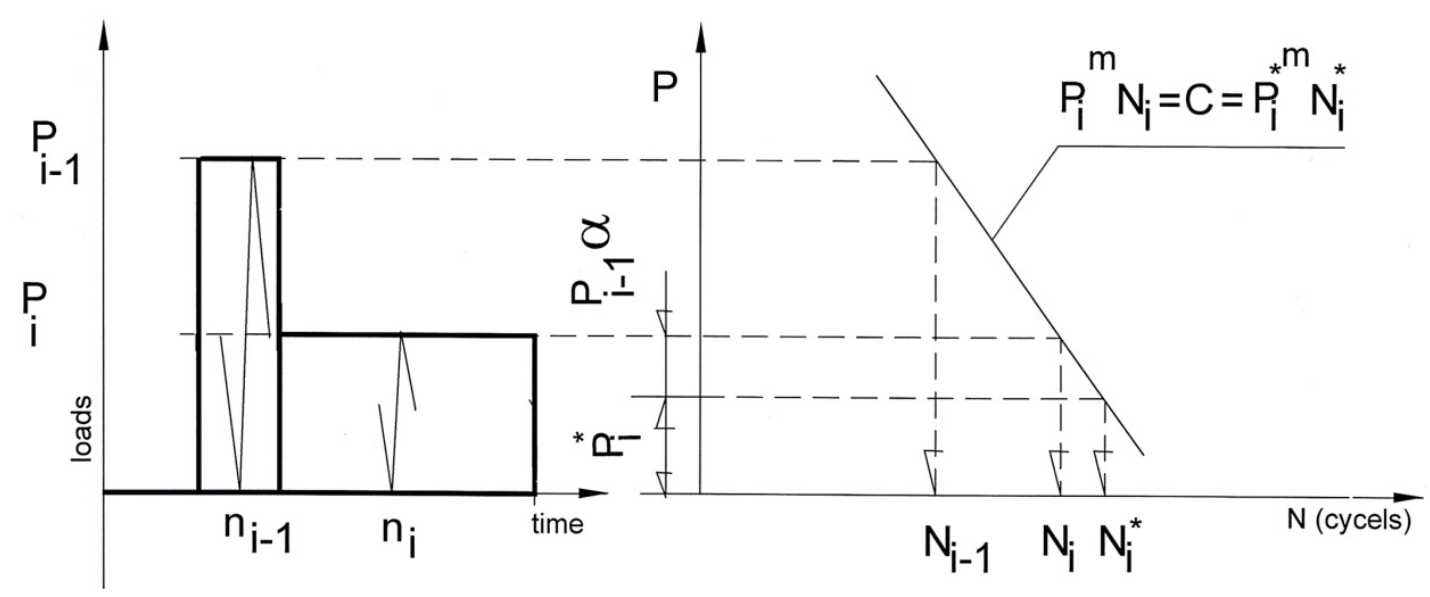

Figure 2. The illustrative presentation of the location of the corrected number of cycles until destruction - $N_{i}^{*}$ 
Then, the proposed criterion of fatigue damage accumulation takes the form:

$$
D_{j}=\frac{n_{1}}{N_{1}}+\sum_{i=2}^{j} \frac{n_{i}}{N_{i}^{*}}
$$

for $j$ - fold sequence - gradual load spectrum. If $\alpha=0$, then we get the Palmgren-Miner hypothesis of linear accumulation of fatigue damage.

Obviously, the number of load cycles $n_{i}$ of the global structure $P_{i}$ is an arbitrarily imposed number of cycles that has been or will be applied to the load-bearing structure. In contrast, the mutual influence of consecutive cycles or sets of load cycles $\left(P_{i-1}\right.$ and $\left.P_{i}\right)$ is shown in the proposed, corrected number of cycles $N_{i}^{*}$ until fatigue failure of the structure.

The computational fatigue analysis of bottom wing plating of a combat trainer aircraft was made as an exemplary application of the hypothesis proposed here (Fig. 4).

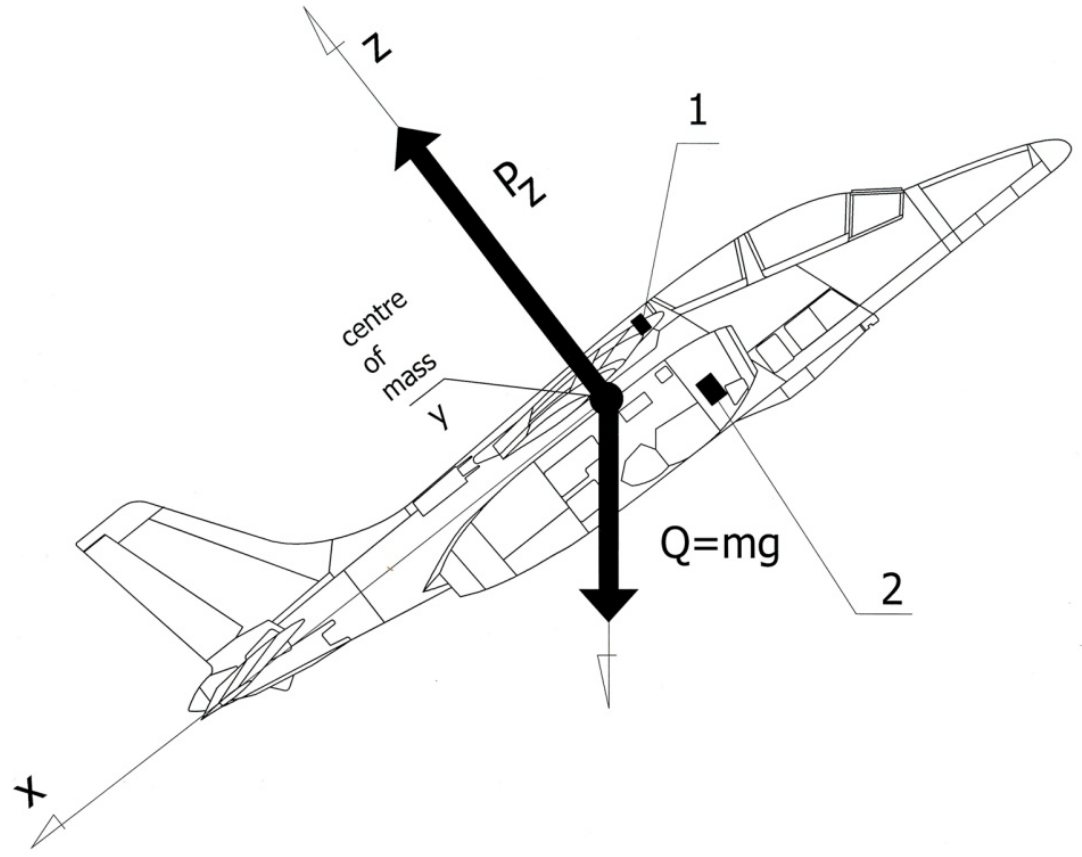

Figure 3. Definition of the aircraft vertical loads coefficient $-n_{z}=P_{z} / Q$,

where: $P_{z}$-aerodynamic lift along the $\mathrm{z}$ - axis, $Q$ - weight of aircraft, $m$ - mass of aircraft, $g$ - gravity acceleration, $x, y, z$ - axis - system of coordinates of aircraft, 1- Aircraft Vertical (Linear) Acceleration Gauge, 2 - Aircraft Vertical Acceleration Sorter

The following assumptions have been made:

- $\quad$ aircraft load spectrum is the same as that adopted in the fatigue test of the wing of PZL I-22 Iryda trainer aircraft (Fig. 5),

- value of the reduced maximum allowable stresses in the analyzed area was set arbitrarily as $\sigma_{\text {dop.max. }}=190 \mathrm{MPa}$, assuming linear relationship between stress in the bottom wing plating and the aircraft load coefficient $n_{z}$ (definition of aircraft vertical loads coefficient $-\mathrm{n}_{\mathrm{z}}$ is presented in Fig. 3),

- fatigue curve was assumed according to [3] Table 2.2.2. It corresponds to duralumin D16T, $\sigma_{0}=100 \mathrm{MPa}$ and the exponent $m=4$, and is described by the equation:

$$
\sigma^{4} N=10^{13}
$$


while for reduction of stress cycle $\left(\sigma_{\max }, \sigma_{\min }\right)$ to repeated (zero - max value) stress cycle $\sigma_{r}$ (for which the cycle fatigue curve is given), the Odgin hypothesis was used:

$$
\left.\sigma_{r}=\sqrt{\sigma_{\max }\left(\sigma_{\max }-\sigma_{\min }\right.}\right)
$$

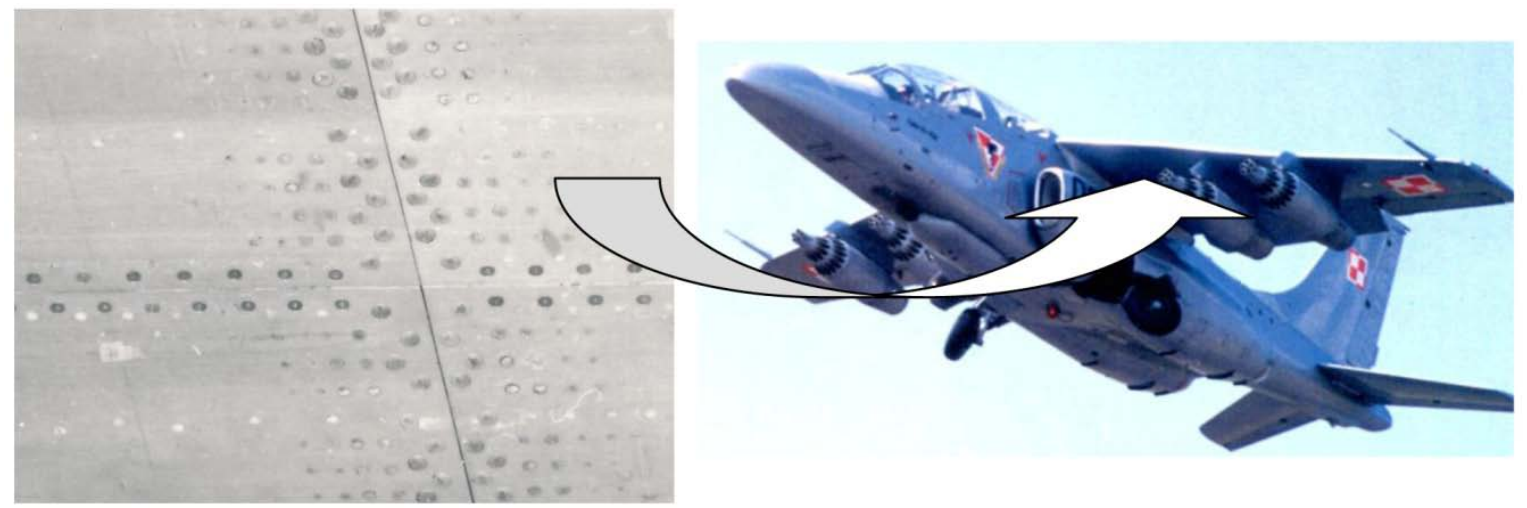

Figure 4. An area of the bottom panels of the aircraft wing selected arbitrarily for the analysis (according to Figure 9.1.2, [3])

The stresses $\sigma_{\text {dop.max. }}$ correspond to the maximum aircraft load coefficient, in this case $n_{z}=8$. For modern aircraft of this class the value of these stresses in the compartment lies within the interval: $150 \mathrm{MPa} \leq \sigma_{\text {dop.max. }} \leq 200 \mathrm{MPa}$. A crucial limitation of this value is not only the fatigue strength, but also resistance to damage, i.e. penetration by bullets, understood as the ability of the structure to transfer the required level of load despite the damage.

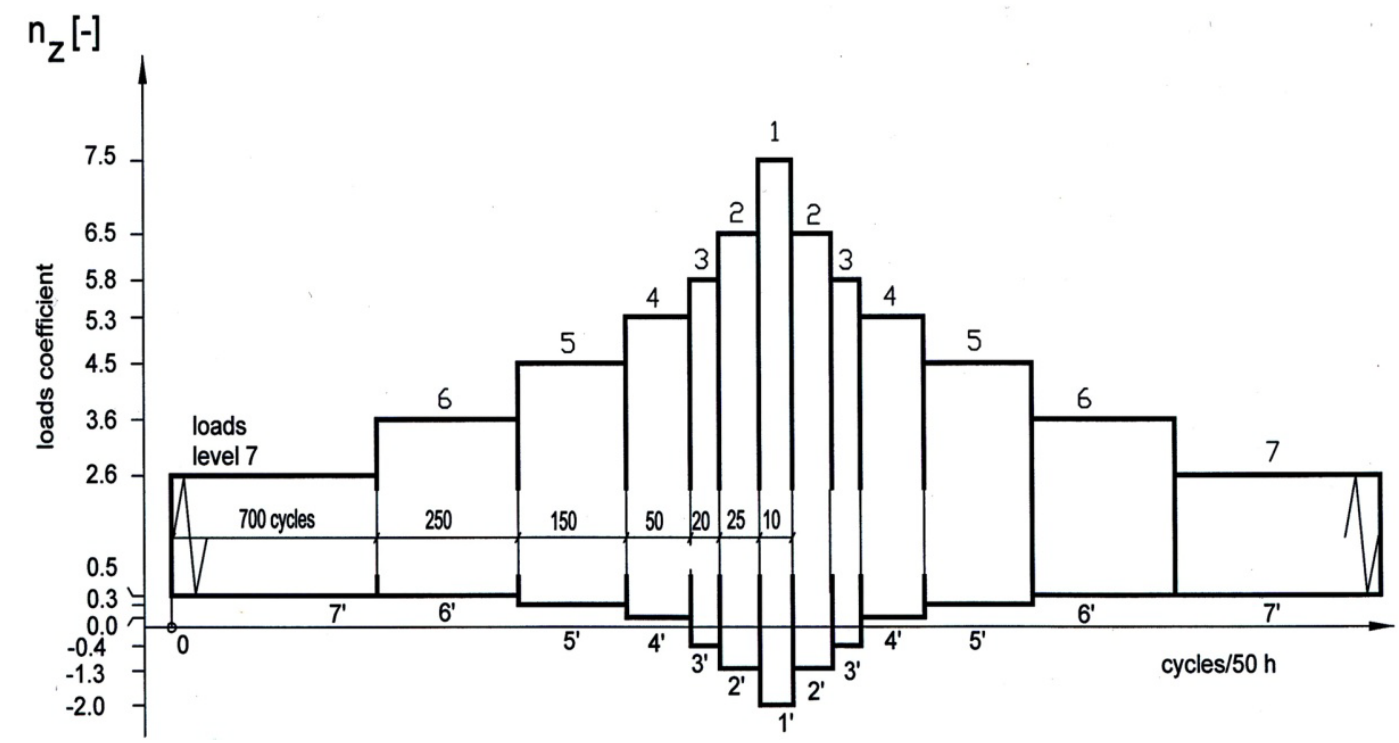

Figure 5. Aircraft load spectrum representing its loads during 50 hours of flight ([3] p. 115) - adopted for the computational analysis

As a result of this analysis, there was obtained and shown in Figure 6 a course of a ratio $\eta=D_{j}$ $(\alpha) / D_{j}(\alpha=0)$ of the value of calculated fatigue damage $D_{j}(\alpha)$ defined by the equation (5) to the fatigue wear $D_{j}(\alpha=0)$ determined by the Palmgren-Miner linear hypothesis of fatigue damage accumulation, depending on the assumed values of the coefficient $\alpha$ and adopted aircraft load spectrum (Figure 5). The estimated fatigue wear values, for the above assumptions, apply to the area of the lower wing panel, shown in Figure 4. 


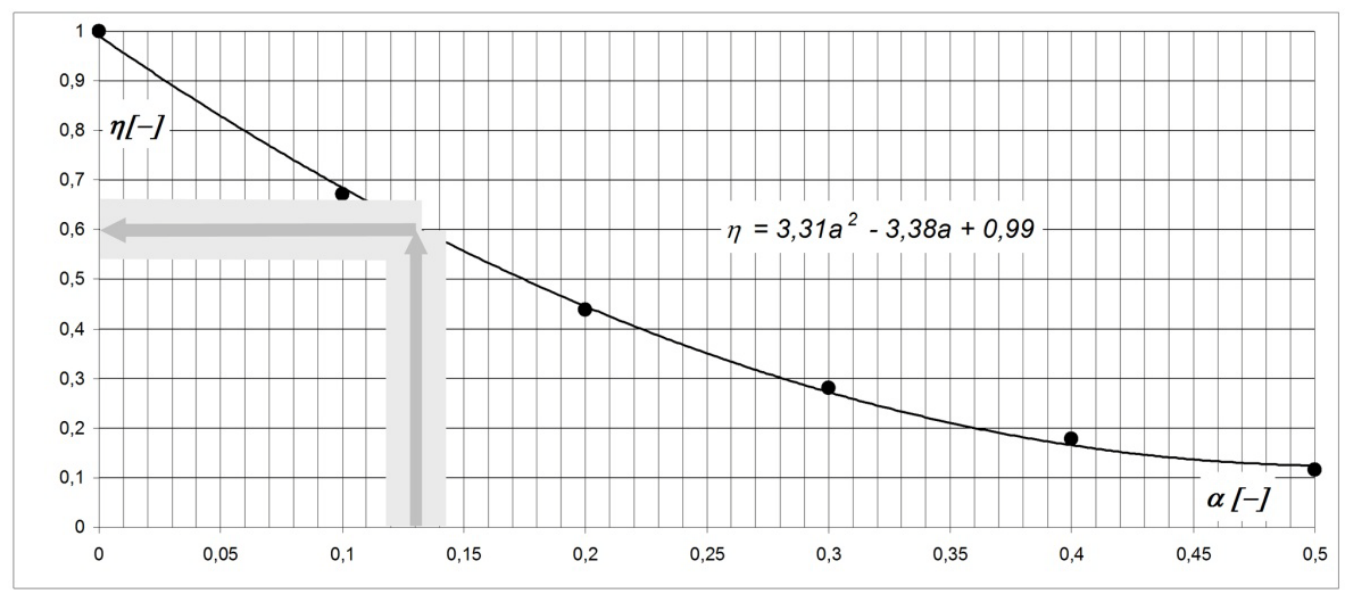

Figure 6. The calculated estimation of fatigue wear $\eta=D_{j}(\alpha) / D_{j}(\alpha=0)$ as a function of the assumed values of coefficient $\alpha$ and its interpolation is indicated by a trend line

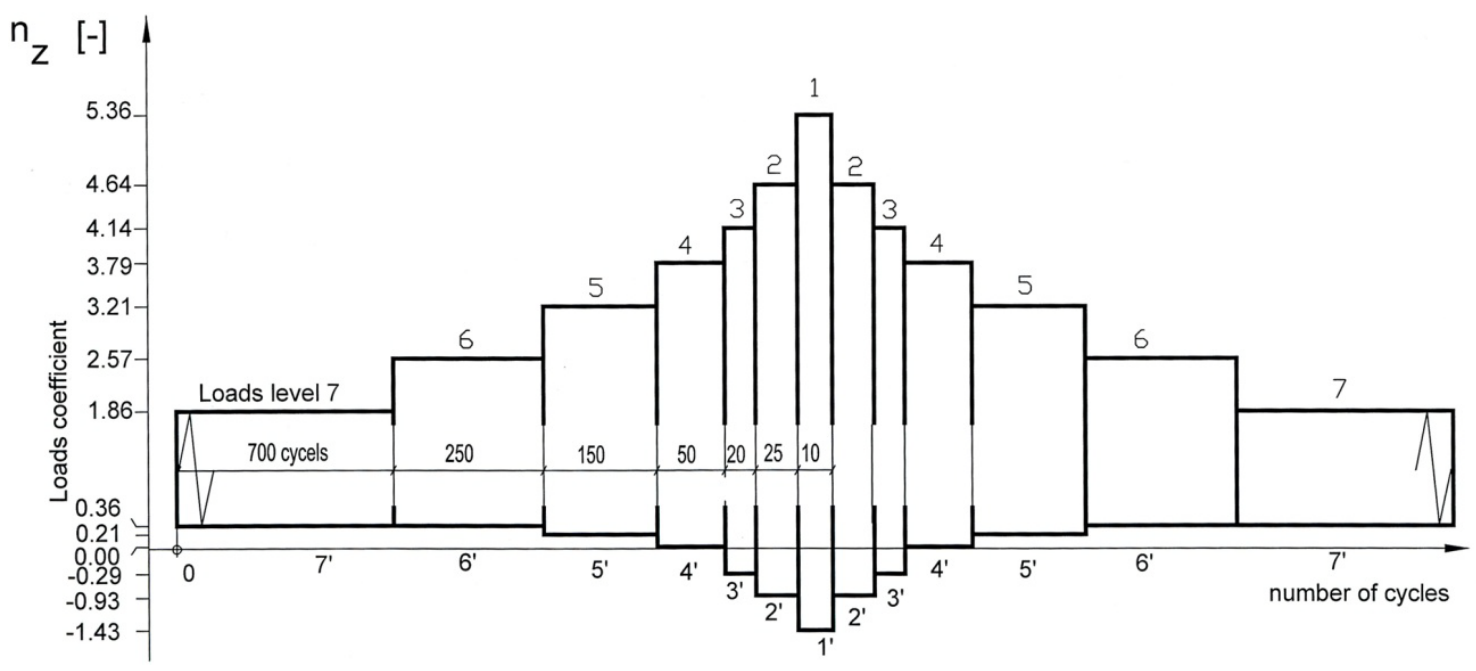

Figure 7. Load spectrum of DEKO-9 Magic aerobatic aircraft assumed for fatigue tests (equivalent 50 flight hours)

\section{CONCLUSIONS}

On the rising side of the spectrum, the proposed criterion gives higher values, while on the sloping side of the spectrum there are lower partial values (for each load level) of fatigue wear in relation to the Palmgren-Miner hypothesis. In all, as we can see from this computational analysis, the adoption of the hypothesis of non-linear accumulation of fatigue damage together with the assumptions made causes nearly a tenfold decrease in the value of this assessment in relation to the linear Palmgren-Miner hypothesis $(\alpha=0)$. In the presented criterion, only two consecutive load sequences are linked by mutual relations, rather than the entire previous load course. This may be a restriction for the use of this hypothesis. A more complex form which takes into account the full load history would create complex formula difficult to use. Perhaps, we should go in this direction, especially that today we have enormous computing power at our disposal.

A detailed discussion of modern hypotheses of fatigue damage accumulation and their assessment in the light of test results was presented by prof. J. Szala in the monograph [4].

The final evaluation of the point and safety of the use of this criterion in the engineering practice could be verified only through fatigue tests and comparison of the results and those of computational fatigue analysis. However, it should be noted that, for heavily loaded supporting 
structures, the computational fatigue analyses serve only to estimate the potential fatigue life at a set load spectrum and stress level, under the condition of not committing significant errors in design or technology.

In order to carry out experimental verification of this hypothesis and, possibly, other hypotheses of fatigue damage accumulation, fatigue tests should be performed, e.g. on cylindrical or tubular (thin-walled) samples with a typical structural notch in the form of a circular hole, made of basic construction materials and examined in complex load conditions (axial force - torsional moment). Tests should be performed for selected load spectrum configurations, so that the impact of the order, size and number of cycles on accumulation of damage could be assessed, in accordance with chosen hypotheses. An example of fatigue tests conducted on samples of this type would be fatigue tests performed at the Warsaw University of Technology [5], except that their aim was to assess the fatigue equivalence of loads, instead of assessing fatigue damage accumulation.

Nowadays, fatigue tests are conducted using structure elements made of 30HGSA steel. Load spectrum is appropriate for the aerobatic airplane DEKO-9 Magic (Figure 7). According to preliminary tests the range of coefficient $\alpha$ is $(0.12,0.14)$, which means that the expected range of coefficient $\eta$ is $(0.55,0.65)$ (Figure 6$)$.

\section{REFERENCES}

[1] Fatemi A., Yang L.: Cumulative fatigue damage and life prediction theories: a survey of the state of the art for homogeneous materials. Int. J. of Fatigue, vol. 20, nº 1, pp. 9-34, 1998.

[2] Schijve, J.: Fatigue of structures and materials in the 20th century and the state of the art. Materials Science, vol. 39, nº 3, pp. 307-333, 2003.

[3] Debski M., Debski D.: Selected problems of fatigue strength of aircraft structures. Scientific Library of the Institute of Aviation 36, ISBN 978-83-63539-06-1, Warsaw 2014.

[4] Szala J.: Hypotheses of fatigue damage summation. Academic Publisher of University of Technology and Agriculture, Bydgoszcz 1998.

[5] Debski D.: Evaluation of material effort in selected structural elements with notches in the form of circular holes. Warsaw University of Technology, 2003

[6] Debski D.: Fatigue tests of structure elements for loads spectrum of DEKO-9 Magic aerobatic aircraft. Warsaw University of Technology 2016 (work in course). 